\title{
Experimental Study on Collimation Characteristics of an ECRH System with a $28 \mathrm{GHz} / 50 \mathrm{~kW}$ Gyrotron
}

\author{
Qing Zhou ${ }^{1,2, *}$ \\ ${ }^{1}$ University of Electronic Science and Technology of China, Chengdu, Sichuan, 610054, China \\ ${ }^{2}$ ENN Science and Technology Development Co., Ltd, Langfang, Hebei, 065001, China
}

\begin{abstract}
In this paper, the collimation characteristics of an electron cyclotron resonance heating (ECRH) system with a $28 \mathrm{GHz} / 50 \mathrm{~kW}$ gyrotron in EXL-50 are studied experimentally. Through using laser trackers and designing matching tooling, test errors of horizontal distances and vertical distances between the transmission line and the flange center are all within $1 \mathrm{~mm}$, which are less than $0.4 \%$ and $0.07 \%$ respectively. In addition, online beam spot test with a $150 \mathrm{~ms}$ short pulse from a $28 \mathrm{GHz}$ gyrotron also proves good collimation characteristics of the ECRH system. Finally, spherical plasma is formed through injecting the microwave to the vacuum chamber.
\end{abstract}

\section{Introduction}

EXL-50 is a medium size spherical Tokamak ${ }^{[1]}$ without central solenoid, whose major $R=0.58 \mathrm{~m}$ and minor radius are $r=0.41 m$ and respectively. Electron cyclotron Resonance Heating is currently the only method to start and maintain plasmas. Photo of the ECRH system with a $28 \mathrm{GHz} / 50 \mathrm{~kW}$ gyrotron ${ }^{[2]-[4]}$ in EXL-50 is shown in Figure 1. Figure 2 shows the layout of the ECRH system in EXL-50. The triode magnetron injection gun is used in the $28 \mathrm{GHz} / 50 \mathrm{~kW}$ gyrotron, which achieved $54.8 \mathrm{~kW} / 1 \mathrm{~s}$ and $45.6 \mathrm{~kW} / 30 \mathrm{~s}$ output power.

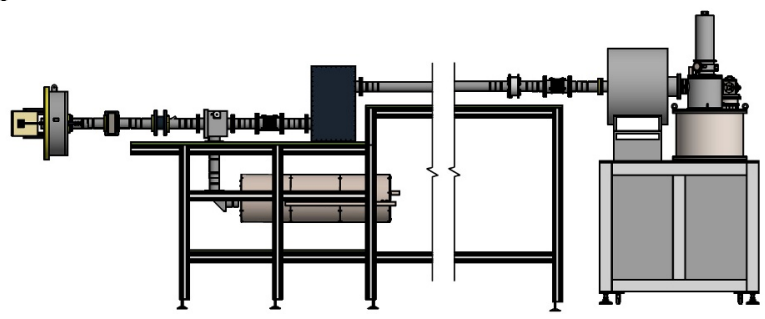

Fig. 1. Photo of the ECRH system with a $28 \mathrm{GHz} / 50 \mathrm{~kW}$ gyrotron in EXL-50

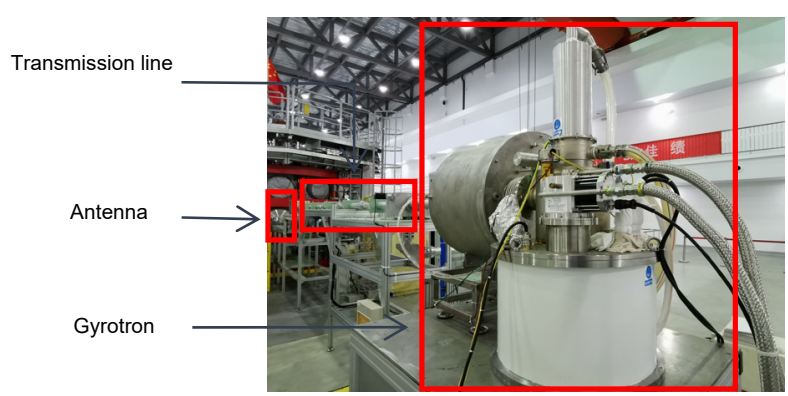

Fig. 2. Layout of the ECRH system in EXL-50
The left and right sides of Figure1 are the antenna and the microwave source of the ECRH system respectively, and the transmission line is between them. The components of the transmission line include straightener, corrugated waveguide, waveguide switch, quasi-light elbow, and the whole distance is about seven meters.

How to ensure collimated transmission of microwaves in the seven-meters transmission line is very important, because non-collimated transmission will cause strong microwave reflection, which in turn will damage transmission components, gyrotron and so on. Therefore, it is particularly significant to study the collimation characteristics of the ECRH system experimentally.

\section{Experimental method and results}

For the accuracy of collimation characteristics experiments, FARO Vantage laser tracker ${ }^{[5]}$ is used, which are extremely accurate, and portable coordinate measuring machines that enable users to build products, optimize processes, and deliver solutions by measuring quickly, simply, and precisely.

A novel matching tooling is designed and machined to cooperate with the use of the laser trackers. Figure 3 (a) is the designed matching tooling with corrugated waveguide, and Figure 3 (b) shows the machined matching tooling with corrugated waveguide and target ball of the laser tracker.

A total of six identical matching toolings have been used in the transmission line of the ECRH system. Five of them are evenly distributed between matching optical unit (MOU) and quasi-light elbow, and the other one is near the antenna.

\footnotetext{
* Corresponding author: zhouqinge@enn.cn
} 


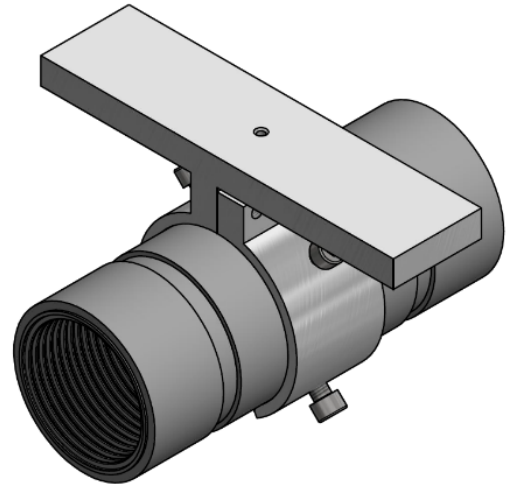

(a)

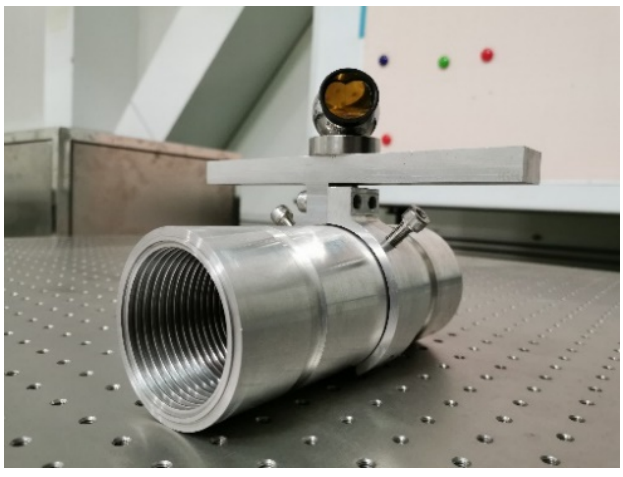

(b)

Fig. 3. (a) The designed matching tooling with corrugated waveguide. (b) The machined matching tooling with corrugated waveguide and target ball of the laser tracker.

Through adjustment by using a laser tracker, horizontal distances between the transmission line and the flange center at six sampling points are acquired and shown in Table 1 (A). Seen from this table, the test errors are within $1 \mathrm{~mm}$, namely less than $0.4 \%$. Similarly, vertical distances between the transmission line and flange center at six sampling points are shown in Table 1 (B). The test errors are also within $1 \mathrm{~mm}$, namely less than $0.07 \%$. It is worth explaining that the height difference between the sixth sampling points and the other five ones comes from the height of quasi-light elbow, and the designed value is $300 \mathrm{~mm}$.

Table 1. (A) Horizontal distances between transmission line and flange center (Units: $\mathrm{mm}$ )

\begin{tabular}{|c|c|}
\hline Element & Value \\
\hline Sampling Points 1 & 102.0 \\
\hline Sampling Points 2 & 102.0 \\
\hline Sampling Points 3 & 102.6 \\
\hline Sampling Points 4 & 102.6 \\
\hline Sampling Points 5 & 102.6 \\
\hline Sampling Points 6 & 102.3 \\
\hline
\end{tabular}

Table 1. (B) Vertical distances between transmission line and flange center (Units: $\mathrm{mm}$ )

\begin{tabular}{|c|c|}
\hline Element & Value \\
\hline Sampling Points 1 & 380.0 \\
\hline Sampling Points 2 & 380.0 \\
\hline Sampling Points 3 & 379.8 \\
\hline Sampling Points 4 & 380.2 \\
\hline Sampling Points 5 & 380.3 \\
\hline Sampling Points 6 & 80.0 \\
\hline
\end{tabular}

To verify the collimation characteristics of the transmission line, the online beam spot test with $28 \mathrm{GHz} / 50 \mathrm{~kW}$ gyrotron is conducted. The detailed experimental parameters are shown in Table II. The beam spot status can be viewed on thermal papers, which are shown in Figure 4. Figures 4 (a), (b), (c), and (d) are the beam spots at a distance of $200 \mathrm{~mm}$ along the corrugated waveguide from MOU, $700 \mathrm{~mm}$ from MOU, $1700 \mathrm{~mm}$ from MOU and the beam spot after microwave passes through the quasi-light elbow, respectively.

Table 2. The detailed experimental parameters used in beam spot test

\begin{tabular}{|c|c|}
\hline Parameters & Value \\
\hline Cathode voltage & $-34 \mathrm{kV}$ \\
\hline Anode voltage & $+1 \mathrm{kV}$ \\
\hline Control pole voltage & $-6 \mathrm{kV}$ \\
\hline Excitation current & $44.3 \mathrm{~A}$ \\
\hline Filament current & $6.6 \mathrm{~A}$ \\
\hline
\end{tabular}

The bright central regions of the four pictures represent the energy concentration areas, and the black regions are low energy areas. Combing Figures 4 (a), (b), (c) and (d), the conclusion could be drawn that the microwave is collimated transmitted, which also proves good collimation characteristics of the ECRH system.

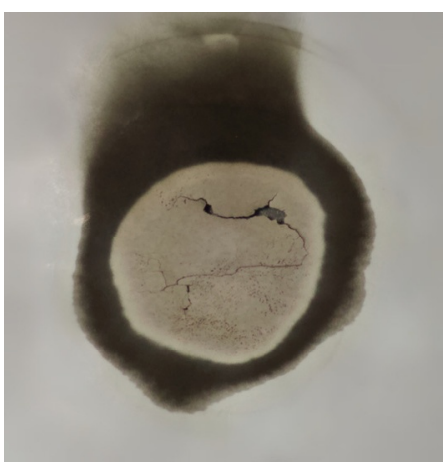

(a) 


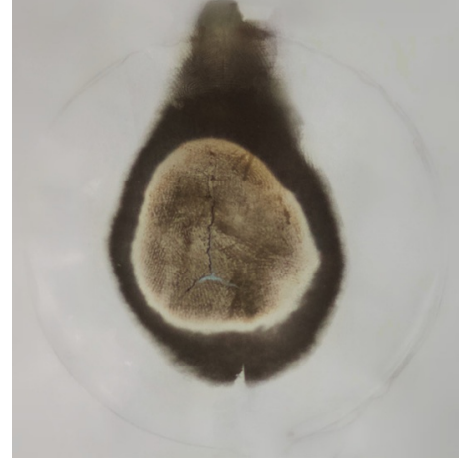

(b)

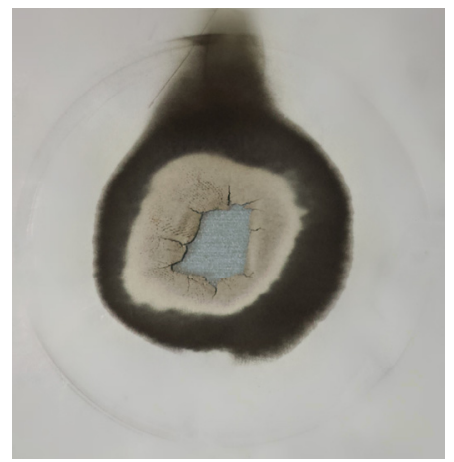

(c)

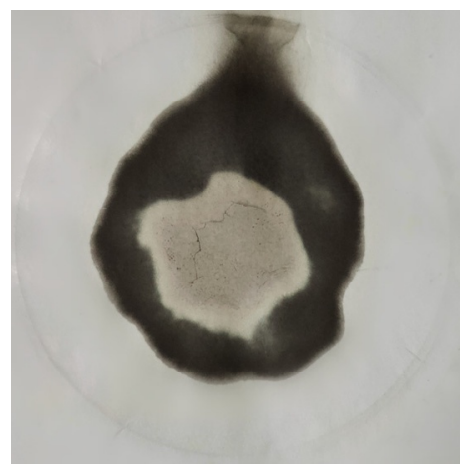

(d)

Fig. 4. (a) The beam spot at a distance of $200 \mathrm{~mm}$ along the corrugated waveguide from the MOU. (b) The beam spot at a distance of $700 \mathrm{~mm}$ along the corrugated waveguide from the MOU. (c) The beam spot at a distance of $1700 \mathrm{~mm}$ along the corrugated waveguide from the MOU. (d) The beam spot after microwave passes through the quasi-light elbow.

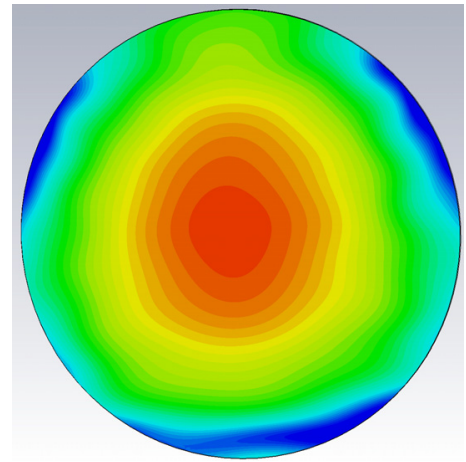

Fig. 5. The simulated energy distribution of Gaussian beam
Figure 5 is the simulated energy distribution of Gaussian beam. Seen from this figure, the red region is high energy area, and with outward expansion, the energy becomes lower and lower. Compared with figure 5 , the photos in figure 4 could be considered as Gaussian beam.

When conducting the online beam spot experiment, the microwave is injected to the water load through waveguide switch. After that, the waveguide switch is switched to the through state so that the microwave is injected to the vacuum chamber. Spherical plasma is formed, which is shown in Figure 6. Figure 6 is the photo of \#4263 from a high-speed camera. During the formation of spherical plasma, spark protection is in normal operation and there is no reflected wave detected.

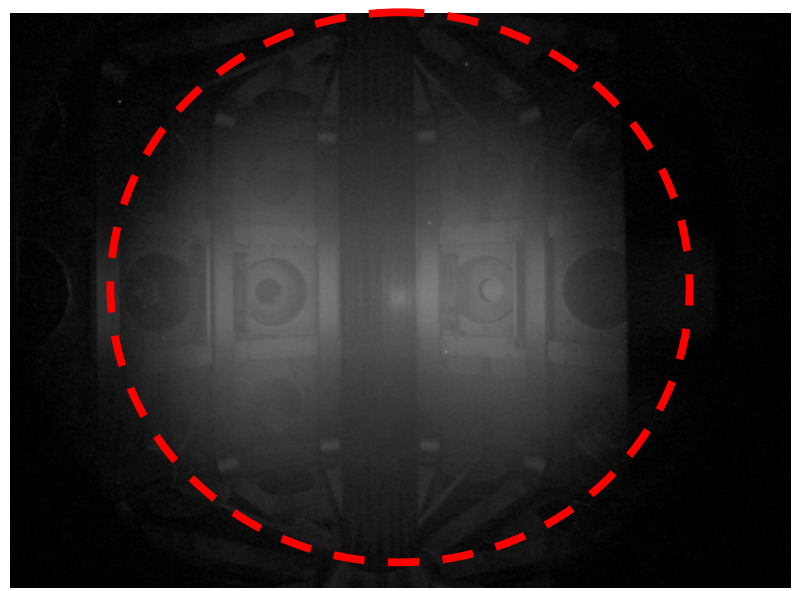

Fig. 6. Photo of \#4263 from a high-speed camera.

In order to obtain higher plasma parameters, the ECRH system with $28 \mathrm{GHz} / 400 \mathrm{~kW}$ gyrotron ${ }^{[6]-[13]}$ is under construction.

\section{Conclusion}

In conclusion, adjusting the collimation characteristics of the ECRH system by designing matching tooling and using laser trackers is pretty good. The test errors of horizontal distances and vertical distances between the transmission line and the flange center are all within $1 \mathrm{~mm}$, which are less than $0.4 \%$ and $0.07 \%$, respectively. Through the online beam spot test with $28 \mathrm{GHz} / 50 \mathrm{~kW}$ gyrotron, the microwave could transfer with good collimation in the transmission line of the ECRH system. In addition, the spherical plasma is obtained after the microwave is injected into the vacuum chamber.

\section{References}

1. Peng, YK M., and Dennis J. Strickler. "Features of spherical torus plasmas." Nuclear Fusion 26.6 (1986): 769.

2. Linlin Hu, Guowu Ma, Dimin Sun, Tingting Zhuo, Qili Huang, Zhiyuan Tan, Shenggang Gong, Hongbin Chen, Fanbao Meng, "A $28 \mathrm{GHz} / 50 \mathrm{~kW}$ continuous wave gyrotron with quasi-optical output," High Power Laser and Particle Beams, vol. 
31, pp. 060101-1 - 060101-2, 2019. doi: 10.11884/HPLPB201931.190139

3. Nusinovich G S, Thumm M K A, Petelin M I. The gyrotron at 50: Historical overview Journal of Infrared, Millimeter, and Terahertz Waves, 2014, 35(4): 325-381.

4. Denisov G. Recent results in IAP/GYCOM development of megawatt gyrotrons. 2018 43rd International Conference on Infrared, Millimeter, and Terahertz Waves (IRMMW-THz). IEEE, 2018: $1-2$.

5. https://www.faro.com/products/3dmanufacturing/faro-laser-tracker-2/]K. Felch, $\mathrm{H}$. Huey, and H. Jory. Gyrotrons for ECH ApplicationsJournal of Fusion Energy, Vol 9, No. 1, 1990.

6. K. Felch, R. Bier, L. Fox , H. Huey ,et al. A 60 $\mathrm{GHz}, 200 \mathrm{~kW} \mathrm{CW}$ gyrotron with a pure outputmode. Int. J. Electronics, 1984, vol. 57, No.6, 815-820.

7. K. Felch, R. Bier, L.J.Craig, et al. CW operation of a $140 \mathrm{GHz}$ gyrotron,Int. J. Electronics, 1986, Vol. 61, No.6, 701-714.

8. Tsuyoshi Kariya. Development of $28 \mathrm{GHz}$ and 77GHz 1MW Gyrotron for ECRH of Magnetically Confined Plasma, Transaction of Fusion Science and Technology,vol.55.

9. Tsuyoshi Kariya, et al. Development of $28 \mathrm{GHz}$ and 77GHz, Mega-Watt Gyrotron for Fusion Devices,J Infrared Milli Terahz Waves (2011) 32:295-310.

10. Tsuyoshi Kariya, et al.Development of $28 \mathrm{GHz}$ gyrotron for cooperative ECH study, Fusion Science and Technology, vol. 68, 2015.

11. Tsuyoshi Kariya, et al.Development of $28 / 35 \mathrm{GHz}$ dual-frequency gyrotron for ECH study,Open Magnetic Systems for Plasma Confinement (OS2016).

12. Tsuyoshi Kariya, et al. Development of over-MW gyrotrons forfusion at $14 \mathrm{GHz}$ to sub-THz frequencies,Nucl. Fusion 57 (2017) 066001 (9pp).

13. Y. Gorelov, J. Lohr, R.W. Callis, and D. Ponce. Infrared Monitoring Of $110 \mathrm{GHz}$ Gyrotron Windows at DIII-D GA Project 30033, May 2002. 